\title{
Engineering Magnetic Domain-Wall Structure in Permalloy Nanowires
}

\begin{abstract}
M. J. Benitez, ${ }^{1}$ M. A. Basith, ${ }^{1, \dagger}$ R. J. Lamb, ${ }^{1}$ D. McGrouther, ${ }^{1}$ S. McFadzean, ${ }^{1}$ D. A. MacLaren, ${ }^{1}$ A. Hrabec, ${ }^{2}$ C. H. Marrows, ${ }^{2}$ and S. McVitie ${ }^{1, *}$

${ }^{1}$ SUPA, School of Physics and Astronomy, University of Glasgow, Glasgow G12 8QQ, United Kingdom

${ }^{2}$ School of Physics and Astronomy, University of Leeds, Leeds LS2 9JT, United Kingdom

(Received 25 July 2014; revised manuscript received 19 December 2014; published 26 March 2015)

Using a focused-ion-beam microscope, we create nontopographic features that provide controlled modification of domain-wall structure, size, and pinning strength in 500-nm-wide nanowires composed from $\mathrm{Cr}(3 \mathrm{~nm}) /$ permalloy $(10 \mathrm{~nm}) / \mathrm{Cr}(5 \mathrm{~nm})$. The pinning sites consist of linear defects where magnetic properties are modified by a $\mathrm{Ga}^{+}$-ion probe of diameter $\sim 10 \mathrm{~nm}$. Detailed studies of the structural, chemical, and magnetic changes induced by the irradiation, which show the modified region to be $\sim 40-50 \mathrm{~nm}$ wide, are performed using scanning-transmission-electron-microscopy modes of bright-field imaging, electron-energy-loss spectroscopy, and differential-phase-contrast imaging on an aberration corrected (Cs) instrument. The Fresnel mode of Lorentz-transmission-electron microscopy is used for studies of domain-wall behavior, where we observe changes in depinning strength and structure with irradiation dose and line orientation. We present an understanding of this behavior based upon micromagnetic simulation of the irradiated defects and their effect on the energy terms for the domain walls.
\end{abstract}

DOI: 10.1103/PhysRevApplied.3.034008

\section{INTRODUCTION}

The controlled manipulation of magnetic domain walls (DWs) in laterally constrained ferromagnets is of huge interest for applications in logic devices [1], spin oscillators [2], and data storage [3]. The domain-wall structure in soft magnetic materials is determined by the width and thickness of the nanowire [4,5] (usually $<20 \mathrm{~nm}$ for the thickness and $<500 \mathrm{~nm}$ for the width). The most commonly investigated soft material, permalloy $\left(\mathrm{Ni}_{80} \mathrm{Fe}_{20}\right)$, has well-studied domain-wall structures, the transverse wall (TW) and vortex wall (VW), which are extended objects, having dimensions comparable with the width of the wire [4]. Considerable attention has been devoted to fabricating in those nanowires geometric modifications such as notches and antinotches of different geometries to control not only the DW structure but also the DW pinning strength at the modified sites [6-9].

Using localized $\mathrm{Ga}^{+}$-ion irradiation, we have previously demonstrated that DWs could be reproducibly pinned at nontopographic sites created by irradiation [10] of a trilayer nanowire of $\mathrm{Cr} /$ permalloy $(\mathrm{Py}) / \mathrm{Cr}$ and that the pinning strength strongly depended on the irradiation dose used. In this article, we extend this investigation, demonstrating that it is possible to select the type, chirality, and size of

\footnotetext{
*stephen.mcvitie@glasgow.ac.uk

†Present address: Department of Physics, Bangladesh University of Engineering and Technology, Dhaka-1000, Bangladesh.

Published by the American Physical Society under the terms of the Creative Commons Attribution 3.0 License. Further distribution of this work must maintain attribution to the author(s) and the published article's title, journal citation, and DOI.
}

the DW in Py nanowires at the nontopographic sites created by $\mathrm{Ga}^{+}$-ion irradiation. A $\mathrm{Ga}^{+}$-ion probe with full width at half-maximum (FWHM) diameter $\sim 10 \mathrm{~nm}$ in the focused-ion-beam (FIB) microscope is used to intermix the layers such that $\mathrm{Cr}$ becomes distributed within the Py layer [11]. Small additions of $\mathrm{Cr}$ are known to significantly reduce the saturation magnetization and Curie temperature of ferromagnetic Ni-Fe alloys, which become paramagnetic at room temperature with only about 8 at. \% $\mathrm{Cr}[11,12]$. Furthermore, we show that the localized changes of the order (2-3) xdiameter of the focused-ion spot can be measured using the differential-phase-contrast (DPC) imaging technique on an aberration-corrected (Cs) scanningtransmission-electron microscope (STEM). Use of this instrument and technique provides the capability of quantitative measurements with nanometer spatial resolution [13]. Using the Fresnel mode of Lorentz microscopy and micromagnetic simulations, we study the local changes in domain-wall behavior for four different line orientations, i.e., $30^{\circ}, 45^{\circ}, 67.5^{\circ}$, and $112.5^{\circ}$ with respect to the wire length and five irradiation doses of $d \times 10^{15}$ ions $/ \mathrm{cm}^{2}$ $(d=4,8,12,16$, and 20). The irradiation dose is chosen taking into account the results from the dynamic ionirradiation simulation package TRIDYN [14] (see the Supplemental Material [15]). These simulations show that 8 at. $\% \mathrm{Cr}$ alloyed into the $\mathrm{Ni}-\mathrm{Fe}$ layer is achieved by an ion dose of $16 \times 10^{15}$ ions $/ \mathrm{cm}^{2}$ [15]. Thus, ferromagnetism is expected to vanish and the material becomes paramagnetic at this dose.

This article is organized as follows. In Sec. II, we describe the experimental details of the material and techniques used, as well as the parameters of micromagnetic simulations. In 
Sec. III, we discuss the results obtained in this work. We start by exploring the structural and magnetic modification of large areas, with width $1 \mu \mathrm{m}$ and length $10 \mu \mathrm{m}$, of thin film caused by $\mathrm{Ga}^{+}$-ion irradiation. Next, we concentrate on the effect of the irradiation on the structural and magnetic properties for a single line written with a $\mathrm{Ga}^{+}$probe of 10 -nm diameter. This local modification is proven to be an efficient method to stabilize DW structures (that are quite different from the equilibrium structure that one would find free in the nanowire) and to control DW chirality and pinning strength. Finally, Sec. IV contains the summary and the conclusions of the study.

\section{EXPERIMENTAL DETAILS}

A thin-film stack of $\mathrm{Cr}(3 \mathrm{~nm}) / \operatorname{Py}(10 \mathrm{~nm}) / \mathrm{Cr}(5 \mathrm{~nm})$ is grown using de sputtering in Ar plasma on top of an electron-transparent $\mathrm{Si}_{3} \mathrm{~N}_{4}$ window substrate suitable for transmission-electron-microscopy (TEM) studies [16]. Nanowires are fabricated from the continuous films by milling the film stack using a FEI Nova NanoLab 200 scanning-electron microscope and focused-ion-beam workstation using a $30-\mathrm{keV} \mathrm{Ga}^{+}$beam energy. The nanowires of $15 \mu \mathrm{m}$ length and $500 \mathrm{~nm}$ width connected to a right-angled bend are shown schematically in Fig. 1(a). Linear defects are created using the FIB with an ion-beam current of $10 \mathrm{pA}$ and an ion-probe size of about $10 \mathrm{~nm}$ in diameter. Nominally, the ion-irradiation doses $(d)$ chosen are $d \times 10^{15}$ ions $/ \mathrm{cm}^{2}$
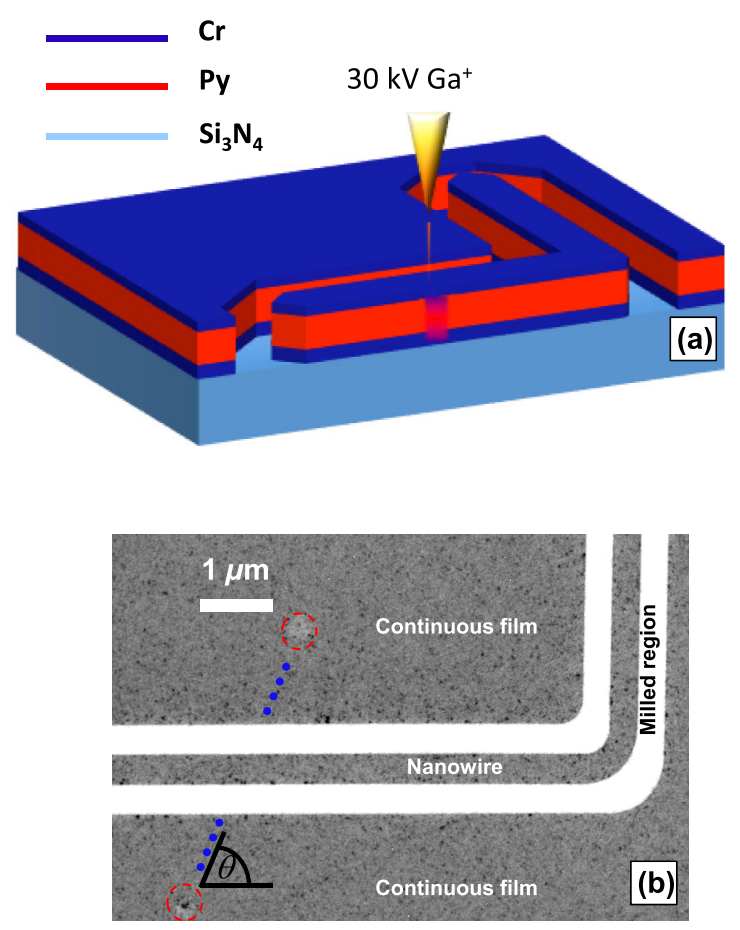

FIG. 1. (a) Schematic of the fabrication process. (b) Plan-view TEM image of the nanowire fabricated by FIB milling. The blue dotted line shows the orientation of the irradiated line which is written continuously between the two red open circles. $\theta$ is the angle between the irradiated line and the wire length. $(d=4,8,12,16$, and 20), which should cause a range of intermixing of the $\mathrm{Cr}$ and Py layers. This localized irradiated single line is oriented at angles of $30^{\circ}, 45^{\circ}, 67.5^{\circ}$, or $112.5^{\circ}$ with respect to the wire length and is defined using the different $\mathrm{Ga}^{+}$doses. Figure 1(b) shows a plan-view TEM image of the nanowire with the irradiated line (highlighted in dotted blue) written at an angle of $67.5^{\circ}$. Note that the line is written between the two heavily irradiated marker spots of diameter $300 \mathrm{~nm}$ that are highlighted in the figure, which help to identify the line's location.

The modification of the magnetic properties of the stack caused by the irradiation is investigated on a continuous film by creating an irradiated stripe pattern $1 \mu \mathrm{m}$ wide and $10 \mu \mathrm{m}$ in length, employing the dose values noted earlier. The stripe patterns are created systematically across the continuous film on top of the $\mathrm{Si}_{3} \mathrm{~N}_{4}$ window membrane and analyzed using bright-field and diffraction imaging as shown in Sec. III.

Next, cross-sectional TEM samples containing irradiated lines of the different doses are prepared using a FIB-based in situ lift-out technique $[17,18]$ to observe the details of the physical structure and chemical compositional of the irradiated areas using electron-energy-loss spectroscopy (EELS). A comparison of the composition of irradiated versus nonirradiated areas allows a confirmation of the composition change induced by the $\mathrm{Ga}^{+}$beam that could be correlated with magnetic measurements. The effect of the magnetically modified line on the domain-wall pinning is investigated using the DPC and Fresnel modes of Lorentz microscopy [19]. Fresnel images and low-angle electron diffraction (LAD) patterns are obtained using a Philips CM20 microscope equipped with a field-emission gun and designed for in situ magnetization experiments, whereas DPC and EELS measurements are obtained using a JEOL ARM-200FCS aberration-corrected (Cs) scanning microscope, both operated at $200 \mathrm{kV}$. EELS data are collected using a Gatan Quantum 965 spectrometer.

To investigate the energetics of domain-wall structures and assist the interpretation of experimental observations obtained by Lorentz microscopy, micromagnetic simulations are carried out using the object-oriented micromagnetic framework (OOMMF) code [20]. Nanowires $500 \mathrm{~nm}$ wide and $10 \mathrm{~nm}$ thick are simulated to contain a modified region of $50 \mathrm{~nm}$ width oriented at $30^{\circ}, 45^{\circ}, 60^{\circ}$, and $120^{\circ}$. The width of the modified region is chosen to take into account the experimental results from cross-sectional TEM imaging of the irradiated region. The simulation cell size is $5 \times 5 \times 5 \mathrm{~nm}^{3}$ for all simulations. The parameters used here are the standard for Py, i.e., the saturation magnetization $M_{s}=8.6 \times 10^{5} \mathrm{~A} / \mathrm{m}$, exchange constant $A=13 \times 10^{-12} \mathrm{~J} / \mathrm{m}$, and zero magnetic anisotropy constant. The saturation magnetization of the modified region $\left(M_{s i}\right)$ is varied from $90 \%$ to $0 \%$ of the standard Py value. The damping coefficient $\alpha$ is set to 0.5 in order to quickly converge the static magnetic structure. 


\section{RESULTS AND DISCUSSION}

\section{A. Effects of the irradiation on the thin film}

Figure 2 shows plan-view TEM bright-field images of (a) the as-deposited unirradiated continuous multilayer film and (b) a region of alternating stripes of unirradiated and $(1-\mu \mathrm{m}$-wide $)$ irradiated material. The irradiated region can be distinguished from the unirradiated film by the increase in grain size, from $5-10 \mathrm{~nm}$ in the unirradiated film to $20-30 \mathrm{~nm}$ in the irradiated region, which is consistent with previous reported studies $[21,22]$.

Quantitative measurements of the magnetic changes due to irradiation in the stripes are obtained using LAD by direct observation of the magnetic structure in the diffraction pattern. LAD manifests as a small-angle splitting of the central diffraction spot due to the change in magnetic induction across a DW in the sample. It may be interpreted classically as a spectrum of the Lorentz deflection angles and magnitudes present in the region of specimen under
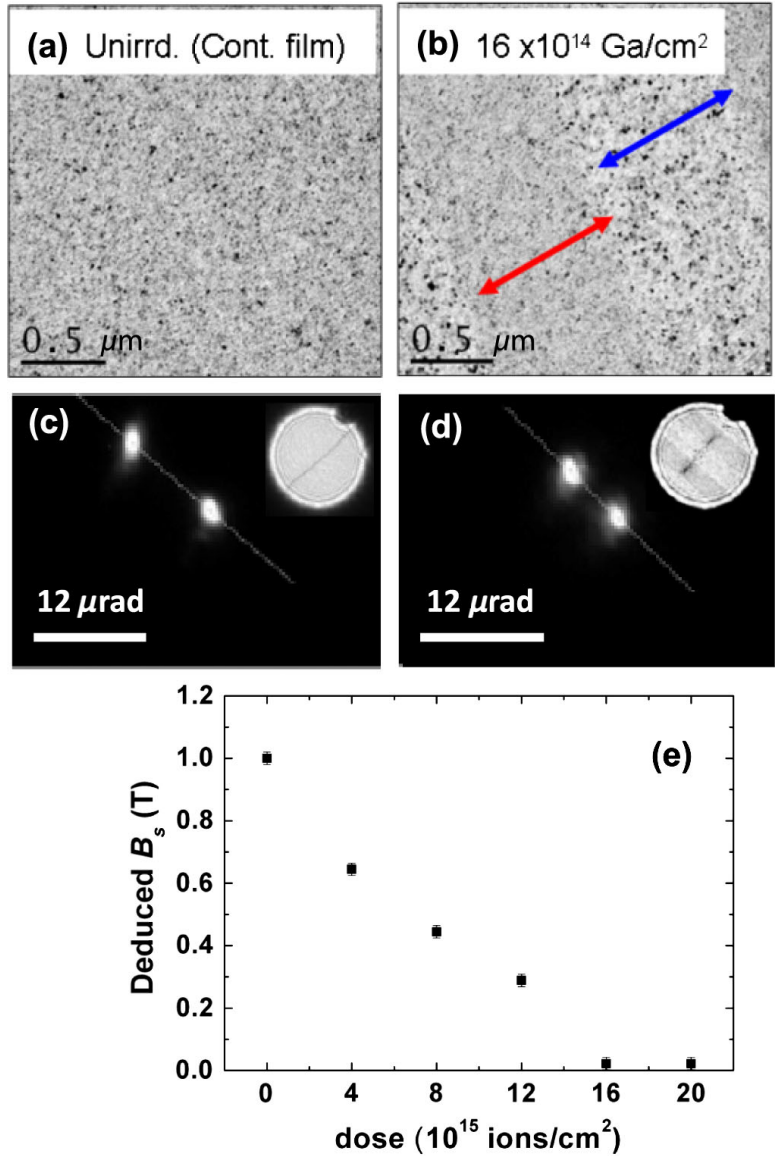

FIG. 2. TEM bright-field images of the (a) unirradiated continuous film and (b) alternating unirradiated and irradiated stripe patterns, indicated by red and blue arrows, respectively. Diffraction patterns from (c) unirradiated continuous film and (d) irradiated regions $\left(16 \times 10^{15}\right.$ ions $\left./ \mathrm{cm}^{2}\right)$. Insets in (c) and (d) show the Fresnel image of a $180^{\circ}$ domain wall in a continuous thin film. (e) Deduced $B_{s}$ as a function of the irradiation ion doses. investigation in TEM. The Lorentz deflection angle $\beta$ of electrons in magnetic materials is given by $\beta_{L}=\left(e \lambda B_{s} t\right) / h$, where $e$ is the electronic charge, $h$ is Planck's constant, $t$ is the film thickness, $B_{s}$ is the saturation induction, and $\lambda$ is the electron wavelength $[19,23]$. This calculation assumes that the magnetization, and hence induction, lie in the plane of the film, perpendicular to the incident electron beam. For 20-nm-thick Py film $\left(B_{s}=1 \mathrm{~T}\right)$ and $200 \mathrm{kV}$ accelerating voltage (i.e., $\lambda=2.5 \mathrm{pm}$ ), $\beta$ is $12.7 \mu \mathrm{rad}$, which is several orders of magnitude smaller than the smallest Bragg diffraction angle of the crystalline lattice.

Low-angle diffraction patterns taken from the films, where a $180^{\circ}$ domain wall is nucleated, are recorded for the unirradiated region [Fig. 2(c)] and from regions modified by different irradiation doses. Figure 2(d) shows the diffraction pattern for an irradiation dose of $16 \times 10^{15}$ ions $/ \mathrm{cm}^{2}$. One observes that the separation between the two diffraction spots is smaller for the deflection which takes place from a region containing irradiated stripes compared to that of the unirradiated region, confirming the reduction of magnetization. By measuring the separation of the spots and using the equation $\beta_{L}=\left(e \lambda B_{s} t\right) / h$, the value of $B_{s}$ can be quantitatively determined and thus the effect of the irradiation deduced. Figure 2(e) shows how the deduced $B_{s}$ decreases as a function of ion dose for the irradiated stripe pattern. As can be seen from this graph, the values range from $1 \mathrm{~T}$ for the unirradiated film to $0 \mathrm{~T}$ for a dose of $16 \times 10^{15}$ ions $/ \mathrm{cm}^{2}$, indicating that the material has been rendered nonferromagnetic at this dose, in agreement with TRIDYN simulations.

\section{B. Effects of the localized irradiation}

The effect of the irradiation of a single line written with a probe of $10-\mathrm{nm}$ diameter is first investigated using a cross-sectional TEM sample. Figure 3(a) shows a dark-field STEM image of the cross section, with contrast deriving principally from atomic number so that the protective $\mathrm{Pt}$ capping layer deposited in the FIB system appears brightest. There is insufficient contrast to distinguish Py from $\mathrm{Cr}$, although the layers are readily observed by EELS, as described below. A small depression in the upper $\mathrm{Cr}$ layer (indicated by the dashed green arrow) indicates the irradiated region, here created with an ion dose of $16 \times 10^{15}$ ions $/ \mathrm{cm}^{2}$. The depression is consistent with a little sputtering and reduction of film thickness in the area of irradiation, in agreement with previous work [21]. Note that the apparent width of the irradiated region is approximately $50 \mathrm{~nm}$, which is clearly larger than the 10-nm ionprobe diameter. This broadening can be understood by considering the means by which the incident ions undergo energy loss in the material. The dominant mode of energy loss for the $30-\mathrm{keV} \mathrm{Ga}^{+}$ions occurs through causing displacements of atoms in the sample. In these interactions, the displaced atoms can possess excess kinetic energy so 
(a)

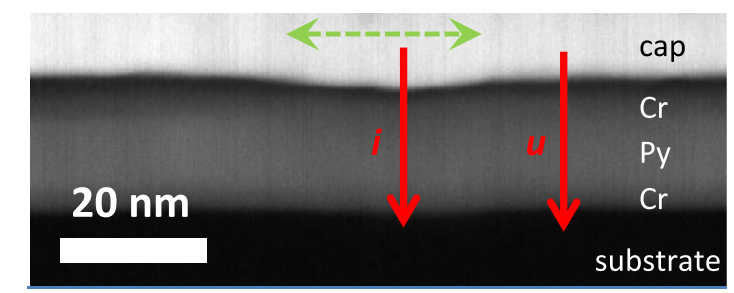

(b)

(c)

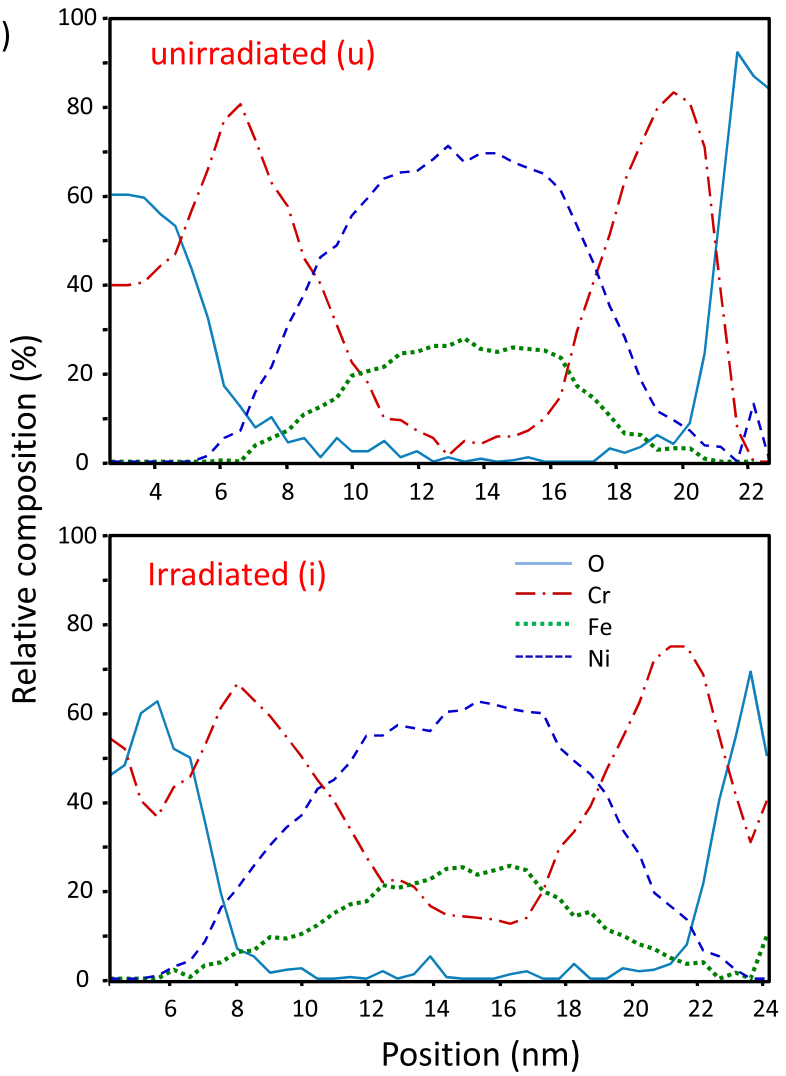

FIG. 3. (a) High-angle annular dark-field (HAADF) STEM image of cross section through an irradiated line in the $\mathrm{Cr}-\mathrm{Py}-\mathrm{Cr}$ stack. The ion dose in the irradiated region is $16 \times 10^{15}$ ions $/ \mathrm{cm}^{2}$ and a protective $\mathrm{Pt}$ capping layer is deposited prior to crosssection fabrication. The green arrows indicate the irradiated zone while the red arrows indicate the location of EELS line scans. (b),(c) Elemental distributions derived from the EELS data and collected from unirradiated $(u)$ and irradiated $(i)$ regions, respectively.

that they travel in random directions, eventually colliding with other atoms in the sample. Thus, a region of altered material is created whose width is larger than the beam diameter and whose overall dimensions depend on factors such as incident beam energy, material density, and average atomic number. Irradiation-induced intermixing of the $\mathrm{Cr}$ and Py layers is assessed by EELS, as summarized in Figs. 3(b) and 3(c), which show the elemental distributions within unirradiated 3(b) and irradiated 3(c) regions. The data are collected using the spectrum imaging technique [24], where an EELS spectrum is acquired pixel by pixel along the direction indicated by the red arrows in Fig. 3(a).
The (normalized) EELS spectral intensity of the $\mathrm{Cr}$, Fe, and Ni $L_{2,3}$ excitation edges are plotted, following removal of a power-law background and assuming standard scattering cross sections. The intensity of the $\mathrm{O} K$ edge is also collected to check for oxidation effects. Processing is performed using the GATAN DIGITAL MICROGRAPH software package. The Cr-Py-Cr stack is apparent in both Figs. 3(b) and 3(c), with negligible oxygen within the stack. None of the layers has a top-hat profile and the tails of the distributions overlap, suggesting a degree of intermixing of $\mathrm{Cr}$ and $\mathrm{Py}$ even in the unirradiated region. However, apparent overlap of the distributions will also occur due to broadening of the scattered electron beam and (more importantly) because the data derive from a projection of a three-dimensionally rough interface onto a twodimensional plane, as described elsewhere [25]. We therefore concentrate on the comparison between the two line scans, which shows a clear difference in the $\mathrm{Cr}$ distribution. Most notably, $\mathrm{Cr}$ is evident throughout the stack in the irradiated region and the $\mathrm{Cr}$ component does not dip below $10 \%$ atomic fraction. This finding suggests that the irradiated region at this dose is paramagnetic at room temperature, consistent with bulk measurements of alloying of Py and Cr [11]. Therefore the EELS data confirm the LAD measurements in Fig. 2(e): that when the film is irradiated with a dose of $16 \times 10^{15}$ ions $/ \mathrm{cm}^{2}$ and above, it becomes paramagnetic and loses its spontaneous magnetization.

Quantitative local measurements of the magnetic properties of the linear defects are explored by DPC imaging. In comparison to $\mathrm{LAD}$, which provides global magnetic information from a relatively wide illuminated region, DPC imaging yields local integrated-induction measurements at very high spatial resolution, below $10 \mathrm{~nm}[13,26]$. DPC measurements are performed on the irradiated lines written between the nanowire edge and one heavily irradiated marked spot (Fig. 1b). The sample is uniformly magnetized by applying a magnetic field parallel to the long axis of the nanowire. Figures 4(a) and 4(b) show the experimental components of induction of an irradiated line at $45^{\circ}$ orientation written with a dose of $8 \times 10^{15}$ ions $/ \mathrm{cm}^{2}$. The DPC detector is aligned so that the components of the induction mapped are orthogonal and parallel to the length of the irradiated line, as indicated by the doubleheaded white arrows. One can clearly observe a contrast change in the irradiated line compared to outside this region in the component mapping along the line [Fig. 4(a)]. This contrast reflects the local difference in magnetic induction. Mapping the component of induction perpendicular to the line, Fig. 4(b), shows no contrast variation. Such a result is entirely expected because the normal component of magnetic induction is continuous across a boundary, in keeping with the requirement for $\nabla \cdot \mathbf{B}=0 . \Delta \beta_{L}$ is measured by taking the difference of the mean value of $\beta_{L}$ of the unirradiated region and the irradiated region across an 

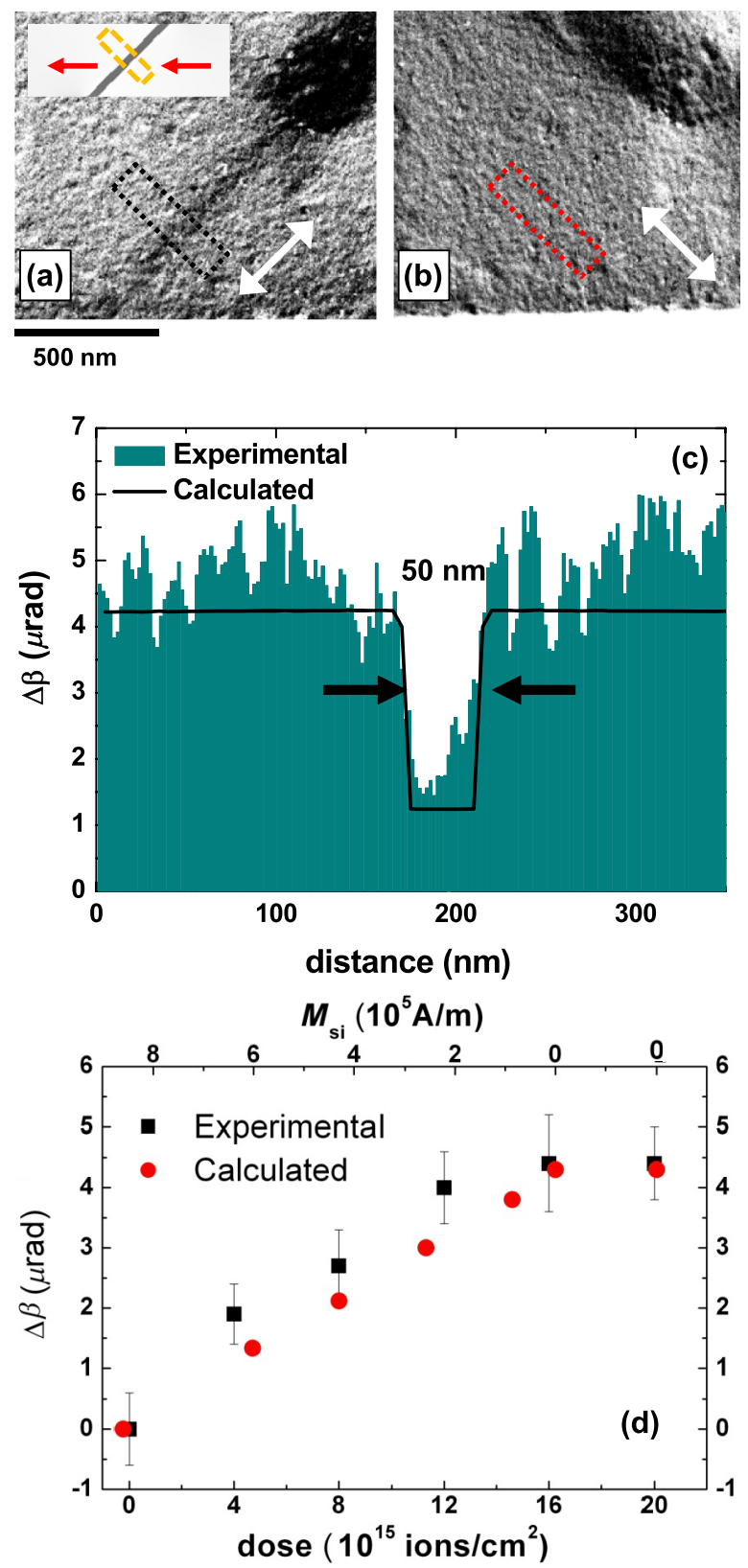

FIG. 4. (a),(b) Experimental DPC images from a linear pinning site of $45^{\circ}$ orientation and irradiation dose of $8 \times 10^{15}$ ions $/ \mathrm{cm}^{2}$. The double-headed white arrows indicate the direction of sensitivity of the mapped induction components. The inset shows calculated Lorentz DPC images from a linear pinning site of $45^{\circ}$ orientation and $M_{s i}=0.3 M_{s}$. (c) Profiles taken from experimental (black dotted rectangle) and calculated images (yellow dotted rectangle) shown on (a). (d) Experimental and calculated $\Delta \beta_{L}$ vs the irradiation dose and $M_{s i}$. Error bars correspond to the standard deviation of $\Delta \beta_{L}$.

area of $400 \times 100 \mathrm{~nm}^{2}$ as indicated by the black dotted rectangle in Fig. 4(a). The profiles taken from the experimental and calculated images are shown in Fig. 4(c), whereas the $\Delta \beta_{L}$ measurements for all different irradiation doses are displayed in Fig. 4(d). The high standard deviation in $\Delta \beta_{L}$ in the experimental results arises due to diffraction effects from the individual crystallites of which the film is composed, which scatter electron intensity into Bragg diffraction channels and therefore affect the intensity of the undiffracted beam. This diffractive contrast has been reduced as much as possible by averaging over the area indicated in Fig. 4(a). We have assumed that the magnetization in the irradiated region is parallel to the length of the wire (the sample is saturated by applying a large field along the wire edge and then reducing this field to zero) and, comparing the experimental and calculated images, we can conclude that for the irradiation doses of $16 \times 10^{15}$ ions $/ \mathrm{cm}^{2}$ and $20 \times 10^{15}$ ions $/ \mathrm{cm}^{2}$, there is enough $\mathrm{Cr}$ alloyed into the $\mathrm{Ni}-\mathrm{Fe}$-alloy film to change the ferromagnetic region to a paramagnetic one. This observation is consistent with the results obtained using LAD and EELS; however, it shows that the local magnetic induction in the line has been quantitatively measured rather than a large-area modified region, which is deduced from the LAD measurements in Fig. 2. Furthermore, the magnetization profile of the irradiated line is seen to have a width of around $50 \mathrm{~nm}$, consistent with the HAADF crosssection image [Fig. 3(a)].

\section{Observed domain-wall behavior}

The magnetic behavior of the DWs pinned at linear defect regions with different orientations and saturation magnetization is now explored. First, a head-to-head TW is created at the bend in the structure, as shown in Fig. 5(a) [with a schematic shown in Fig. 5(b)]. The initial TW is obtained by applying a magnetic field of around 600 Oe along the wire length and relaxing the field to zero. Next, a magnetic field of opposite sign is applied along the wire length to propagate the DW towards the modified region. The propagation field, i.e., the field required to move the DW from the corner position to the pinning site, is identical for all wires, being $8 \pm 2$ Oe as reported previously [10]. Figures 5(c) and 5(d) show Fresnel images of the DW structure for linear-pinning irradiated sites written with a dose of $4 \times 10^{15}$ ions $/ \mathrm{cm}^{2}$ and with two different orientations, i.e., $30^{\circ}$ and $67.5^{\circ}$. A head-to-head TW is observed in both cases. Note that the leading part of the TW is pinned along the line written at an angle of $67.5^{\circ}$, whereas the wall is just pinned at one point of the pinning site, oriented at $30^{\circ}$. Included in Figs. 5(e) and 5(f) are Fresnel images calculated from simulations where head-to-head DWs are artificially introduced in wires with local property modifications at two different orientations, $30^{\circ}$ and $60^{\circ}$ and with $M_{s i}=0.9 M_{s}$. The red arrows in the calculated images depict the direction of the magnetization around the defect. The calculated Fresnel image, Fig. 5(e), also demonstrates that the leading part of the TW is pinned just where the line meets the lower edge of the wire as observed in the experiment, Fig. 5(c). For a line orientation of $60^{\circ}$ [calculated Fresnel image, Fig. 5(f)], the leading part of the wall is 

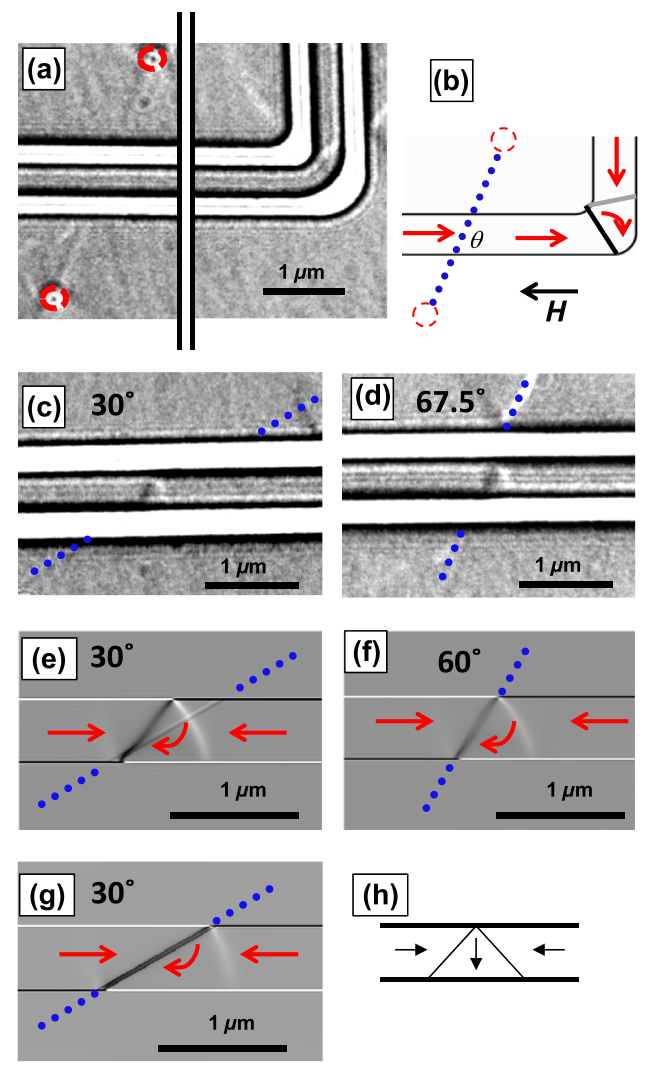

FIG. 5. (a),(c),(d) Fresnel images of $\mathrm{Cr} / \mathrm{Py} / \mathrm{Cr}$ nanowires. (a) TW at zero field located at the bend in the nanowire and (b) corresponding schematics. (c),(d) DWs pinned at the linear irradiated sites, written with a dose of $4 \times 10^{15}$ ions $/ \mathrm{cm}^{2}$ but with different orientations. (e)-(g) Calculated Fresnel images from simulations of the wires with local property modifications with $M_{s i}=0.9 M_{s}(\mathrm{e}),(\mathrm{f})$ and $M_{s i}=0.8 M_{s}(\mathrm{~g})$. The blue dotted lines show the orientation of the irradiated lines whereas the red arrows represent the magnetization direction. Note that TW is pinned just where the line meets the lower edge of the wire for (c) and (e), whereas for $(\mathrm{d}),(\mathrm{f}),(\mathrm{g})$, the leading part of the wall is pinned along the defect. (h) Schematic of a TW in a uniform part of a nanowire.

pinned along the defect, as is observed in the experimental Fresnel image, Fig. 5(d). By reducing the magnetization in the defect, i.e., for $M_{s i} \leq 0.8 M_{s}$, the DW is pinned along the modified region of the nanowire oriented at $30^{\circ}$, as seen in the simulated image, Fig. $5(\mathrm{~g})$.

Experimentally, a further reduction of magnetization at the modified region can be achieved by increasing the irradiation dose. Thus, irradiation doses of $d \times 10^{15}$ ions $/ \mathrm{cm}^{2}$ (with $d=8,12$, and 16) are applied for all the linear orientations. Figures 6(a), 6(b), and 6(c) show Fresnel images of DWs pinned at the linear irradiated sites written with a dose of $16 \times 10^{15}$ ions $/ \mathrm{cm}^{2}$. As the irradiation dose increases, there is a change of the DW structure and VW becomes the preferred wall type, independent of the line orientation. The observed DW for higher doses with linear orientation smaller than $90^{\circ}$ is always a VW with a clockwise sense of circulation, or
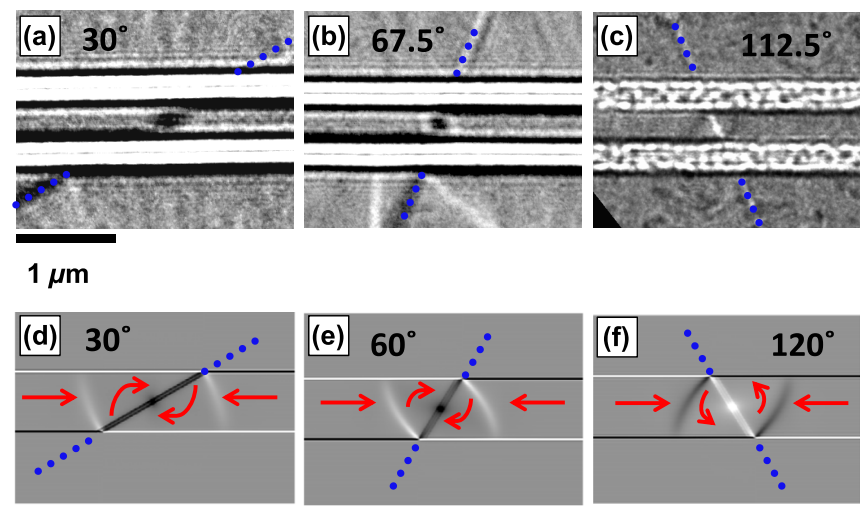

FIG. 6. (a)-(c) Fresnel images of $\mathrm{Cr} / \mathrm{Py} / \mathrm{Cr}$ nanowires showing DWs pinned at the linear irradiated sites written with a dose of $16 \times 10^{15}$ ions $/ \mathrm{cm}^{2}$. (d)-(f) Corresponding calculated Fresnel images from simulations.

chirality. This observation is in agreement with previous observations [10], where only a $45^{\circ}$ linear defect is investigated. In this case, the central wall section of the VW aligns along the defect. For the cases observed here, this wall section appears to show the wall extended [Fig. 6(a), 30 ${ }^{\circ}$ ] and compressed [Fig. 6(b), 67.5 ], which is consistent with the central wall section aligning with the defect in each case. Counterclockwise VWs are obtained for wires where the linear defect is written at an angle higher than $90^{\circ}$, as shown in Fig. 6(c). In this case, for the central wall section to align along the defect requires the opposite-chirality VW compared to those at the lower angle. A simulation of this situation confirms the experimental findings [Figs. 6(d)-6(f)]. Overall, this behavior is attributed to induced asymmetry in the energy landscape of the modified region of nanowire due to ion irradiation. Thus, the preferred chirality of the VW is that one for which the angle between the central wall section and the irradiated line is minimized.

Furthermore, depinning-field $H_{\text {dep }}$ values are measured for different domain-wall configurations. Table I $\left(H_{\mathrm{dep}}\right.$ directions are shown in schematics at the top of the table) summarizes the results for an initial TW structure pointing down and lists the subsequent pinned wall structure and its depinning field as a function of the line dose and line orientation with the statistics included to show the variation in wall type observed at the defect.

\section{Discussion of energetics and of DW behavior}

Consideration of the energetics of domain-wall structure and the influence by the irradiated defects is necessary to explain the observations we make in Sec. III C. For domain walls in straight nanowires, structure and energetics have been the subject of detailed micromagnetic study [4] that yielded a phase diagram predicting the ground-state domain-wall configuration based on wire geometry. Wider, thicker wires tend to favor vortex-type walls, while thinner, 
TABLE I. $\quad H_{\text {dep }}$ as a function of irradiation doses for pinning sites with different orientations of head-to-head DW. In the initial transverse DW structure, the magnetization points down $(\downarrow)$ as shown in (a). DW structure at the pinning site is also shown. CVW, clockwise vortex; CCVW, counterclockwise vortex. The table shows results from twelve measurements performed on each wire. Each measurement starts from the state shown in (a) and then follows the propagation and pinning (b), and depinning (c).

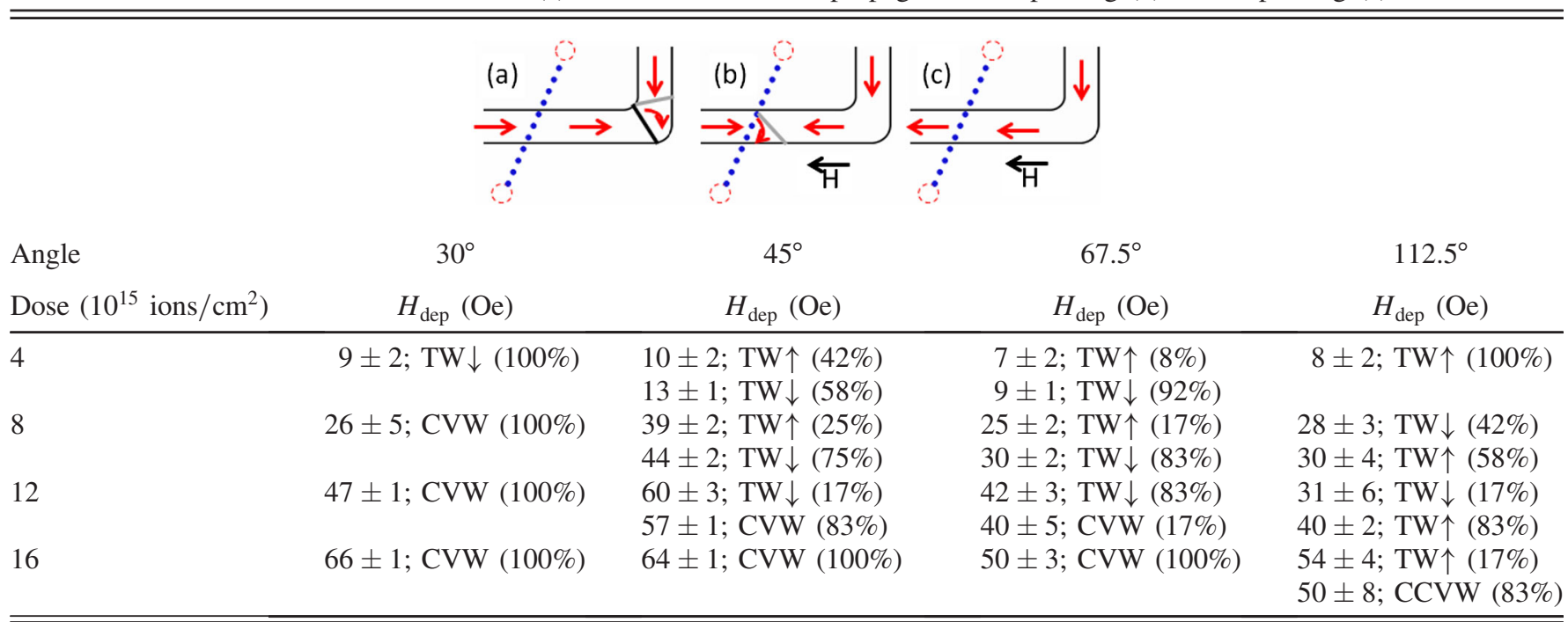

narrower wires favor transverse walls. For the wire dimensions employed here, the expected ground energy state would be a vortex wall. In all of our experiments, we have produced an initial transverse (down) domain wall through the use of a polarizing field at the bend in the wire; see Table I, schematic (a). The metastable transverse wall appears to remain stable in the propagating field although it has been seen to change its structure at the defect as we have observed both here and in our previous study [10].

Guided by the microscopy characterization in Sec. III B, we have conducted extensive micromagnetic simulations to understand the effects of the irradiated defects in pinning

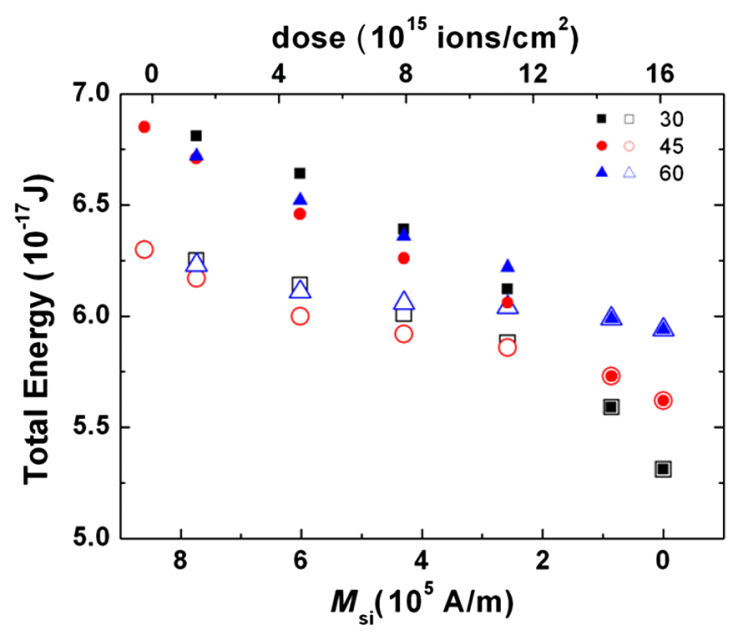

FIG. 7. Calculated total energy vs $M_{s i}$ for different orientations of the modified region, for VW (open symbols), and TW (closed symbols) domain walls in the 500-nm-wide, 10-nm-thick wire. walls and causing alterations to their structure. Figure 7 shows a plot of the total energy for pinned domain walls with respect to the magnetization $M_{s i}$ of the irradiated line defects with $30^{\circ}, 45^{\circ}$, and $60^{\circ}$ angles. In agreement with Ref. [4], at $M_{s i}=8.6 \times 10^{5} \mathrm{~A} / \mathrm{m}$, vortex-type walls are predicted to have $\sim 10 \%$ lower energy than transverse walls for this wire geometry. For both wall types, reducing the local strength of the magnetization $\left(M_{s i}\right.$ decreasing from $8.6 \times 10^{5} \mathrm{~A} / \mathrm{m}$ to zero) in the line defect leads to a monotonic reduction of the total DW energy (reducing both magnetostatic and exchange energy terms) with irradiation dose. Such magnetization strength reduction constitutes the creation of a potential energy well. Any displacement of the wall away from the irradiated defect immediately leads to an increase in the total energy.

From the observations presented in Sec. III C, we have identified a wide range of behavior for defects of different orientations. Taking these results together with our previous study, we can conclude that the overall trend is an increase in depinning field with dose for each defect angle together with a transformation of the pinned wall from transverse domain wall to vortex walls at higher doses. In Table I, however, we record that more complicated behavior is observed for linear defects irradiated at intermediate doses and we proceed to discuss this behavior in detail.

We start by looking at an example of wall-type changes as a function of angle for the lowest dose. From Table I, a polarizing effect is seen on the initial transverse down wall [Fig. 8(a)] at the two extreme angles. In the case of the $30^{\circ}$ irradiated defect, the leading edge of the wall exhibits a similar alignment with the linear defect and, after propagation to the pinning site, the wall remains as a transverse down type [Fig. 8(b)]. By contrast for the $112.5^{\circ}$ defect, 

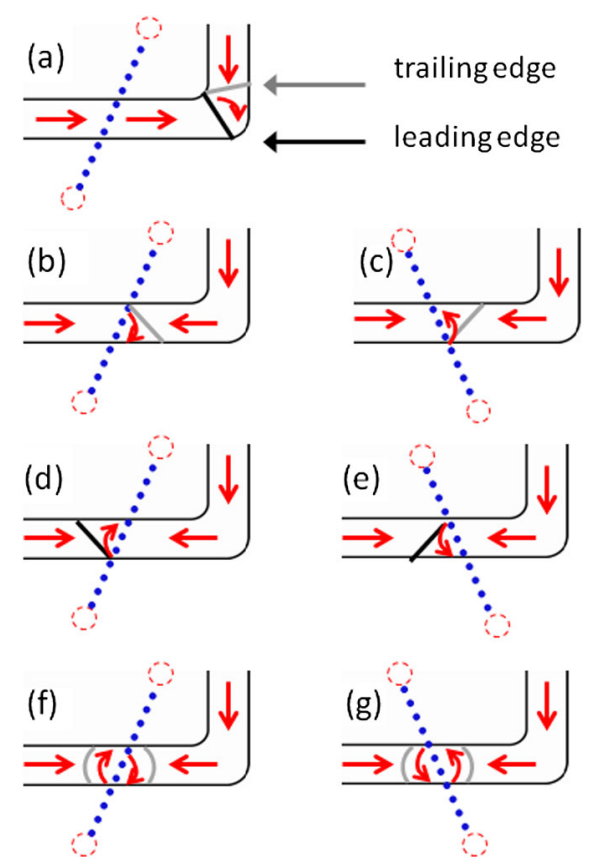

FIG. 8. Schematics of the differing wall geometry. (a) Initial transverse down wall. TWs with leading edge pinned to the defect: (b) down and (c) up. TWs with the trailing edge pinned to the defect: (d) up and (e) down. (f), (g) VWs.

where the leading edge of the down wall is now poorly aligned to the defect (having an almost orthogonal alignment to the defect), the result is that the wall transforms to an up wall in order that the leading edge better matches the defect [Fig. 8(c)]. Between the two extrema, for intermediate angles, a mixture of up and down walls is seen. However, we need to differentiate between the up and down transverse walls and their positions relative to the defect, as shown in Figs. 8(b)-8(e). In terms of the field direction, they can be considered as pinned by their leading [Figs. 8(b) and 8(c)] or trailing [Figs. 8(d) and 8(e)] edges, i.e., the up and down walls are different for line defects with orientation angles above and below $90^{\circ}$. From Table I, in all cases where we observe a mix of transverse walls at a particular dose and angle, we see a small but consistent difference between the depinning field for the up and down walls. What we note is that the depinning field is higher where the leading edge of the wall is pinned at the defect [Figs. 8(b) and 8(c)], whereas it is lower for the trailing edge pinned at the defect [Figs. 8(d) and 8(e)]. Simplistically, this variation makes sense in that the walls with the trailing edge are pulled from the defect whereas those pinned by the leading edge need to be pushed through the defect. Furthermore, the chirality of the vortex domain wall now can be seen to be defined for angles below (CVW) and above $90^{\circ}$ (CCVW) as is observed in Fig. 6. This chirality selection is also illustrated in Figs. 8(f) and $8(\mathrm{~g})$ and is due to the alignment of the middle wall section with the defect line for a given chirality.
Another interesting observation is that the depinning field from the $45^{\circ}$ line appears to be generally higher than for the other angles. Consideration of the total wall energy in Fig. 7 may provide an explanation for such an effect. For walls in nanowires with no defect, the natural relaxed structure of both transverse- and vortex-type DWs favors a $45^{\circ}$ orientation for the respective $90^{\circ}$ (in the transverse-type DW) and $180^{\circ}$ (in the vortex-type DW) Néel wall components that compose the total DW packet. Therefore, with an irradiated defect positioned at this angle, the individual component walls are well matched to the defects. However, in the case with the defects at angles other than $45^{\circ}$, the component walls must distort to align with the defect, suggesting that energy will be significantly higher than for defects at $45^{\circ}$. Figure 7 shows that the total energy of the transverse and vortex DWs is calculated to be lowest for all of the $45^{\circ}$ defects. Thus, the $45^{\circ}$ structure may be regarded as the deepest potential well and requiring a greater applied field strength to extract the DW from this potential.

Finally, we discuss the appearance of vortex walls for higher irradiation doses as recorded in Table I. With increasing irradiation dose, Fig. 7 shows that the energy of TWs decreases more rapidly than for VWs, and for irradiation causing $M_{s i} \leq 0.1 M_{s}$, VW and TW energies become equivalent. In Fig. 9, we show micromagnetic simulations of head-to-head TWs and VWs at the irradiated defect. When $M_{s i}=0.9 M_{s}$, the TW [Fig. 9(a)] and the VW [Fig. 9(b)] exhibit their commonly observed structures. Corresponding to irradiation at a high dose, when $M_{s i}=0.3 M_{s}$, Fig. 9(c) reveals that the simulated structure of the TW becomes considerably modified compared to the usual TW, with the structure becoming more like the VW of Fig. 9(d) but with a significantly reduced transverse component of magnetization. For the modified TW in Fig. 9(c) to become the VW of Fig. 9(d), a vortex core must be nucleated. This is a process that we would expect to occur easily as a result of thermal activation-the vortex being nucleated at the top edge of the wire and immediately
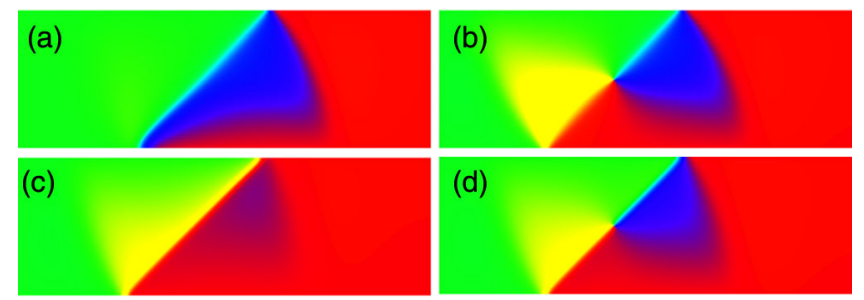

(e)

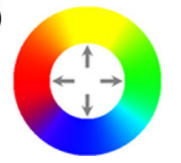

FIG. 9. Calculated color images from simulations of the wires with local property modification. (a) Shows a TW and (b) a VW with $M_{s i}=0.9 M_{s}$. (c) Shows the modified TW and (d) the VW with $M_{s i}=0.3 M_{s}$. (e) Is the color wheel. 
traveling along the $180^{\circ}$ Néel wall line to occupy a central position. Experimentally, the appearance of the vortex wall for higher angle defects $\left(\geq 45^{\circ}\right)$ is observed for irradiation doses $\geq 12 \times 10^{15}$ ions $/ \mathrm{cm}^{2}$, which corresponds to $M_{s i}<$ $0.3 M_{s}$ as shown in Fig. 4(d). The appearance of the vortex wall at much lower dose for the $30^{\circ}$ defect compared to line defects with other orientations is attributed to the fact that an extension of the wall is needed for the transverse wall to align to the defect. Thus, the transformation from the metastable transverse wall to a presumably ground-state vortex structure is more likely for lower doses.

\section{CONCLUSIONS}

In summary, our results demonstrate that the structure of DWs in these permalloy multilayer nanowires has been "engineered" by local property modification via the linear defect region. The angle of the linear defect and/or irradiation dose can serve to stabilize DW structures that are quite different from the equilibrium structure that one would find in an unmodified nanowire. TWs are favored at lower doses and larger defect angles, whereas VWs are predominant at the highest doses and lower angles. By changing the angle of the irradiated line, it is possible to vary the DW packet width, pinning strength, and vortex chirality. Additionally, the observed transverse walls are noted to have different pinning behavior depending on whether they are pinned with their leading or trailing edge at the defect. Quantification of the effects of the $\mathrm{Ga}^{+}$-ion irradiation is made possible using the methods of Lorentz microscopy. In particular, using DPC imaging, we show that it is possible to detect changes in magnetic induction for samples with locally reduced saturation magnetization on a length scale of Néel domain-wall widths (a few tens of nanometers).

\section{ACKNOWLEDGMENTS}

We would like to thank W. A. Smith and C. R. How for their expert technical assistance. This research is supported by the Engineering and Physical Sciences Research Council, EPRSC (Grants No. EP/I013520 and No. EP/ I011668/1), the Scottish Universities Physics Alliance, and the University of Glasgow.

[1] D. A. Allwood, G. Xiong, C. C. Faulkner, D. Atkinson, D. Petit, and R. P. Cowburn, Magnetic domain-wall logic, Science 309, 1688 (2005).

[2] V. S. Pribiag, I. N. Krivorotov, G. D. Fuchs, P. M. Braganca, O. Ozatay, J. C. Sankey, D. C. Ralph, and R. A. Buhrman, Magnetic vortex oscillator driven by d.c. spin-polarized current, Nat. Phys. 3, 498 (2007).

[3] S. S. P. Parkin, M. Hayashi, and L. Thomas, Domain-wall racetrack memory, Science 320, 190 (2008).
[4] Y. Nakatani, A. Thiaville, and J. Miltat, Head-to-head domain walls in soft nano-strips: A refined phase diagram, J. Magn. Magn. Mater. 290, 750 (2005).

[5] M. Laufenberg, D. Backes, W. Bührer, D. Bedau, M. Kläui, U. Rüdiger, C. A. F. Vaz, J. A. C. Bland, L. J. Heyderman, F. Nolting, S. Cherifi, A. Locatelli, R. Belkhou, S. Heune, and E. Bauer, Observation of thermally activated domain wall transformations, Appl. Phys. Lett. 88, 052507 (2006).

[6] A. Vogel, A. C. Niemann, C. Stenner, A. Drews, M.-Y. Im, P. Fischer, and G. Meier, Vortex dynamics in triangularshaped confining potentials, J. Appl. Phys. 112, 063916 (2012).

[7] L. K. Bogart, D. Atkinson, K. O'Shea, D. McGrouther, and S. McVitie, Dependence of domain wall pinning potential landscapes on domain wall chirality and pinning site geometry in planar nanowires, Phys. Rev. B 79, 054414 (2009).

[8] D. Petit, H. T. Zeng, J. Sampaio, E. Lewis, L. O’Brien, A.-V. Jausovec, D. Read, R. P. Cowburn, K. J. O'Shea, S. McVitie, and J. N. Chapman, Magnetic imaging of the pinning mechanism of asymmetric transverse domain walls in ferromagnetic nanowires, Appl. Phys. Lett. 97, 233102 (2010).

[9] W. Zhu, J. Liao, Z. Zhang, B. Ma, Q. Y. Jin, Y. Liu, Z. Huang, X. Hu, A. Ding, J. Wu, and Y. Xu, Depinning of vortex domain walls from an asymmetric notch in a permalloy nanowire, Appl. Phys. Lett. 101, 082402 (2012).

[10] M. A. Basith, S. McVitie, D. McGrouther, and J. N. Chapman, Reproducible domain wall pinning by linear non-topographic features in a ferromagnetic nanowire, Appl. Phys. Lett. 100, 232402 (2012).

[11] R. M. Bozorth, Ferromagnetism (IEEE Press, New York, 2003).

[12] J. Fassbender, J. von Borany, A. Mücklich, K. Potzger, W. Möller, J. McCord, L. Schultz, and R. Mattheis, Structural and magnetic modifications of Cr-implanted Permalloy, Phys. Rev. B 73, 184410 (2006).

[13] J. N. Chapman, I. R. McFadyen, and S. McVitie, Modified differential phase contrast Lorentz microscopy for improved imaging of magnetic structure, IEEE Trans. Magn. 26, 1506 (1990).

[14] W. Möller, W. Eckstein, and J. P. Biersack, TRIDYN-binary collision simulation of atomic collisions and dynamic composition changes in solids, Comput. Phys. Commun. 51, 355 (1988).

[15] See Supplemental Material at http://link.aps.org/ supplemental/10.1103/PhysRevApplied.3.034008 for results of simulation of ion irradiation.

[16] B. Khamsehpour, C. D. W. Wilkinson, J. N. Chapman, and A. B. Johnston, High resolution patterning of thin magnetic films to produce ultrasmall magnetic elements, J. Vac. Sci. Technol. B 14, 3361 (1996).

[17] J. Mayer, L. A. Giannuzzi, T. Kamino, and J. Michael, TEM sample preparation and FIB-induced damage, MRS Bull. 32, 400 (2007).

[18] M. A. Basith, S. McVitie, D. McGrouther, J. N. Chapman, and J. M. R. Weaver, Direct comparison of domain wall behavior in permalloy nanowires patterned by electron beam lithography and focused ion beam milling, J. Appl. Phys. 110, 083904 (2011). 
[19] J. N. Chapman, The investigation of magnetic domain structures in thin foils by electron microscopy, J. Phys. D 17, 623 (1984).

[20] M. J. Donahue and D. G. Porter, NIST Report No. NISTIR 6376, National Institute of Standards and Technology, 1999.

[21] D. Ozkaya, R. M. Langford, W. L. Chan, and A. K. PetfordLong, Effect of Ga implantation on the magnetic properties of permalloy thin films, J. Appl. Phys. 91, 9937 (2002).

[22] D. McGrouther, J. N. Chapman, and F. W. M. Vanhelmont, Effect of $\mathrm{Ga}^{+}$ion irradiation on the structural and magnetic properties of CoFe/IrMn exchange biased bilayers, J. Appl. Phys. 95, 7772 (2004).
[23] K. Takayanagi, T. Koyama, S. Mori, K. Harada, and Y. Togawa, Small-angle electron scattering from magnetic artificial lattice, J. Electron Microsc. 61, 401 (2012).

[24] J. A. Hunt and D. B. Williams, Electron energy-loss spectrum-imaging, Ultramicroscopy 38, 47 (1991).

[25] M. S. Gabureac, D. A. MacLaren, H. Courtois, and C. H. Marrows, Long-ranged magnetic proximity effects in noble metal-doped cobalt probed with spin-dependent tunnelling, New J. Phys. 16, 043008 (2014).

[26] J. N. Chapman and M. R. Scheinfein, Transmission electron microscopies of magnetic microstructures, J. Magn. Magn. Mater. 200, 729 (1999). 